\title{
Real and Modeled Spike Trains: Where Do They Meet?
}

\author{
Vasile V. Moca ${ }^{1}$, Danko Nikolić (2,3 $^{\text {, and Raul C. Mureşan }}{ }^{1,2}$ \\ 1 Center for Cognitive and Neural Studies (Coneural), Str. Cireşilor nr. 29, \\ 400487 Cluj-Napoca, Romania \\ moca@coneural.org, muresan@coneural.org, \\ WWW home page: http://www. coneural.org/muresanlab \\ 2 Max Planck Institute for Brain Research, Deutschordenstrasse 46, \\ 60528 Frankfurt am Main, Germany \\ 3 Frankfurt Institute for Advanced Studies, Ruth-Moufang-Str. 1, \\ 60438 Frankfurt am Main, Germany
}

\begin{abstract}
Spike train models are important for the development and calibration of data analysis methods and for the quantification of certain properties of the data. We study here the properties of a spike train model that can produce both oscillatory and non-oscillatory spike trains, faithfully reproducing the firing statistics of the original spiking data being modeled. Furthermore, using data recorded from cat visual cortex, we show that despite the fact that firing statistics are reproduced, the dynamics of the modeled spike trains are significantly different from their biological counterparts. We conclude that spike train models are difficult to use when studying collective dynamics of neurons and that there is no universal 'recipe' for modeling cortical firing, as the latter can be both very complex and highly variable.
\end{abstract}

\section{Introduction}

The activity of cortical neurons arises from complex firing patterns that are determined by the intricate brain architecture, the external stimuli and the interaction with subcortical structures [1]. Each cortical neuron receives on the order of $10^{3}-10^{4}$ inputs from other neurons, and hence it is prone to being exposed to a high input bombardment [2]. Indeed, it has been suggested that such a bombardment exists, and, in addition to a balanced excitation-inhibition state, it keeps the neuron in a so-called "high-conductance state" [3]. Moreover, under such heavy input, cortical neurons have a tendency to fire highly irregularly, such that the distribution of their inter-spike intervals (ISI) takes an exponential form [4]. As a result, numerous models of spike trains have been proposed [5,6], which assume an underlying homogenous (constant firing probability over time) or inhomogeneous (the instantaneous firing probability can fluctuate over time) Poisson process [7]. Recently however, the heavy input bombardment hypothesis has been challenged [8], new [9] and old [10] data suggesting that the assumption 
that neurons are simple Poisson spike generators is an extreme oversimplification. The firing properties of neurons depend critically on neuron type [11], brain structure [12], brain state [13], arousal [14], and other factors.

A particular case, where firing is non-Poissonian, is represented by the oscillatory discharge of cortical neurons. In such cases, neurons can engage into various rhythms in multiple frequency bands [15]. The oscillatory firing properties of neurons can be characterized by computing auto-correlation histograms $(\mathrm{ACH})$, and we have previously introduced a measure, called Oscillation Score [16] that is useful in determining the degree to which a neuron oscillates. In order to precisely quantify the oscillation strength we needed to develop a model of oscillatory spike trains, in which the oscillatory behavior and the firing rate could be independently controlled [16]. Here, we explicitly study the properties of this model: the precision with which firing rate and oscillation strength can be controlled and the ability to independently express the two properties in the simulated spike trains. In addition, we are interested in the degree to which artificial spike trains, generated from statistical parameter distributions of recorded neuronal data, reflect the temporal structure of the latter.

\section{The model}

The model produces artificial spike trains that retain basic properties of a recorded data set. The considered properties are: the firing rates, burst probability, oscillation strength and frequency, spike counts in bursts, refractory periods, and intra-burst inter-spike intervals, all of which are quantitatively determined from biological data. Two processes are used to generate the spike trains: at a coarse scale a discharge probability function $p_{s}(t)$ (Fig. 1A, B), and at a finer scale another process (Fig. 1C) that controls the exact spike timings (refractoriness, burst properties, etc). Since our model is not a biophysical one, most of the parameters that we used have no direct biological correspondents.

The spike discharge probability $p_{s}(t)$ should have the following properties: first, it should allow the spike train to exhibit a preferred oscillation frequency for transient periods of time; second, it should allow control over the strength and stability of the desired oscillation; and third, it should enable the control over the firing rates. To control the amount of oscillations, two discharge probabilities $p_{o}(t)$ and $p_{b}(t)$ corresponding respectively to an oscillatory and a background process are intermixed with a factor $o$ (oscillation strength) (1). To obtain the transient oscillatory behavior we modulate the frequency of a sine probability function $p_{o}(t)(2)$ by a random process $f_{o}(t)(4)$. This random process takes into account past values and thus, it has memory. The history dependence is given by a decay constant, $\tau$, while another factor, $m$, controls the amount of noise added to the random process. After a duration of $3 \tau$, a value fades to less than $5 \%$ and its effect is very small. The interplay between $\tau$ and $m$ controls how the oscillation frequency changes over time. The frequency range of the modulatory function, $f_{o}(t)$, can be bounded to increase the stability of the oscillation. It is assumed that prior to $t=0$ the function $f_{o}(t)$ varies slightly 
around the desired oscillation frequency. The background probability $p_{b}(t)(3)$ is generated in the same way except that in this case, the frequency of the process $f_{b}(t)$ (which has the same form as $f_{o}(t)$ ) varies in a much broader frequency range (Fig. 1B). For a spike to be generated, $p_{s}(t)$ must be positive. The greater $p_{s}(t)$, the greater is the chance that a spike will be generated and thus, most of the spikes will be concentrated at the peaks of the probability functions (Fig. $1 \mathrm{C})$. By manipulating the offset (1) we can control how well the spikes are aligned to the desired oscillation (negative values of $p_{s}(t)$ mean that no spike is generated), and by manipulating the spike-probability-positive-integral (SPPI) (5) trough the amplitude parameter, $A(1)$, we control the firing rates (Fig. $1 \mathrm{~A}$ and Fig. 2 - left column) for the duration $T$ of the spike train. Thus, the value of $p_{s}(t)$ controls the periodicity and firing rates of spike-trains in a manner that realistically mimics the oscillatory behavior of the recorded neurons.

$$
\begin{gathered}
p_{s}(t)=A \cdot\left[o \cdot p_{o}(t)+(1-o) \cdot p_{b}(t)-o f f \text { set }\right] \\
p_{o}(t)=\frac{1}{2}+\frac{1}{2} \cdot \sin \left(2 \cdot \pi \cdot \int_{0}^{t} f_{o}(x) d x\right) \\
p_{b}(t)=\frac{1}{2}+\frac{1}{2} \cdot \sin \left(2 \cdot \pi \cdot \int_{0}^{t} f_{b}(x) d x\right) \\
f_{o}(t)=\lim _{\epsilon \rightarrow 0} \frac{\int_{\epsilon}^{3 \tau} e^{-\frac{x}{\tau}} \cdot f_{o}(t-x) d x}{\int_{\epsilon}^{3 \tau} e^{-\frac{x}{\tau}} d x}+m \cdot \operatorname{rand}_{[-1,1]}(t) \\
S P P I=\frac{1}{T} \int_{0}^{T} p_{s}^{+}(t) d t \\
\text { where: } p_{s}^{+}(t)= \begin{cases}p_{s}(t), & p_{s}(t) \geq 0 \\
0, & p_{s}(t)<0\end{cases}
\end{gathered}
$$

At smaller time scales, the model controls the timings between the spikes and the burstiness of the spike trains. The burst probability, $p_{\text {burst }}$, is modeled as a constant ratio between the count of bursts relative to the number of tonic spikes and burst occurrences altogether. These values can be extracted from a recorded data set. Once a discharge is initiated based on, $p_{s}(t), p_{\text {burst }}$ determines whether that discharge will be a burst or tonic spike. If a burst is generated, the number of spikes in the burst $b_{S p k C n t}$ and the spacing between the spikes within the burst $b_{I S I}$ (Fig. 1C) are set according to probabilities measured from real data. After a tonic spike a refractory period, $r$, prevents the occurrences of other discharges in a given period of time. The model makes a clear distinction between the tonic spikes and bursts. The bursts are defined as groups of spikes with successive ISIs smaller or equal to $8 \mathrm{~ms}$ [17]. Thus, the refractory period, $r$, is set to $8 \mathrm{~ms}$ such that tonic spikes can occur with an ISI of at least $9 \mathrm{~ms}$. The control over these timings at a small scale produces a realistic center-peak shape in the ACH (Fig. 3) of artificial spike trains which model real spike trains. 


\section{A}
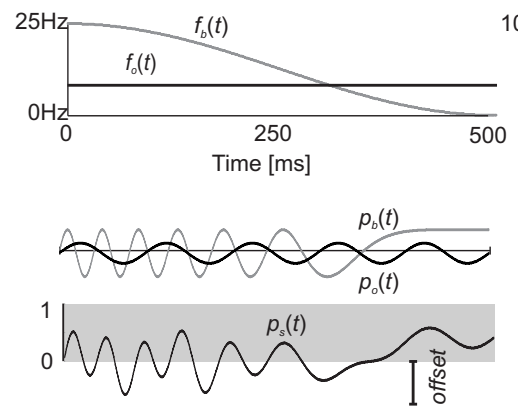

B

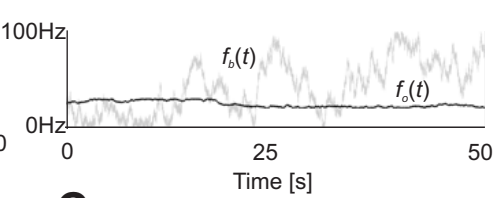

C

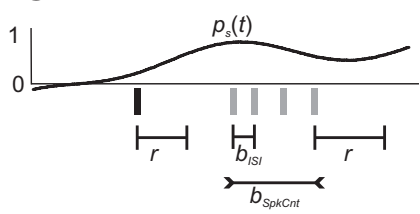

Fig. 1. Model for simulation of oscillatory spiking activity. A: Spike discharge probability. The spike discharge probability $p_{s}(t)$ is obtained by mixing two time-varying processes $p_{b}(t)$ and $p_{o}(t)$ with frequencies $f_{b}(t)$ and $f_{o}(t)$, respectively and with a mixing factor $o=0.3$ (see Eq. 1). The amplitude and offset of the two processes are fixed for one run of the algorithm. A spike can be generated only where $p_{s}(t)$ is positive (grey band). B: Fluctuations of the modulation functions $f_{b}(t)$ and $f_{o}(t)$. While $f_{b}(t)$ varies between 0 and $100 \mathrm{~Hz}$ (gray) $f_{o}(t)$ varies slowly around $25 \mathrm{~Hz}$ due to its strong history dependence and boundaries $(20$ to $30 \mathrm{~Hz})$. C: Spiking. A burst of spikes is represented by grey vertical bars, while a tonic spike is depicted in black. The spikes are generated taking into account the refractory period after a burst or a tonic spike $r$, the intra-burst inter-spike interval $b_{I S I}$ and the burst spike count $b_{\text {SpkCnt }}$.

\section{Results}

We addressed here two important aspects related to the model: the relationship between firing rate and oscillation strength, and the degree to which the model can reproduce the fine temporal structure of recorded spike trains.

\subsection{Firing rate and oscillation strength}

We computed the relationship between firing rate and oscillation strength by producing artificial spike trains, with a length of $30 \mathrm{~s}$, and controlling independently the two parameters for rate and oscillation strength. The rate parameter (SPPI; see Methods) and the oscillation strength parameter $(o)$ were varied in 24 steps. For each combination, two spike trains were produced, yielding a total of 1152 spike trains. In addition, we generated such spike trains for each oscillation frequency band separately: theta $(4-8 \mathrm{~Hz})$, alpha $(8-12 \mathrm{~Hz})$, beta-low $(12-20 \mathrm{~Hz})$, beta-high $(20-30 \mathrm{~Hz})$ and gamma $(30-80 \mathrm{~Hz})$ [16]. For the oscillation frequency in the model $\left(f_{o}\right)$, we took the central (middle) frequency for each frequency band. The firing rate and the oscillation score [16] (the measured strength of oscillation) were measured for each spike train. We found that, the firing rate could be more precisely controlled for higher than for lower frequency bands (Fig. 2, left column, note the lower variance for higher frequency 
bands) while the oscillation score seemed to be controlled equally well across all frequency bands (Fig. 2, middle column). Moreover, the firing rate scaled as a power function $\left(R^{2}>0.93\right)$ with respect to the $S P P I$ while the oscillation score scaled as a sigmoid with respect to the oscillation strength parameter. The noisy clouds in Fig. 2 (middle column), that deviate from the sigmoid shape are due to trials with very small firing rates, for which the oscillation score estimate becomes imprecise [16]. Finally, there was no correlation between the firing rate and oscillation score, as shown in Fig. 2, right column $\left(R^{2}<0.08\right)$. Thus, the resulting firing rate and oscillation strength can be independently controlled by the two corresponding parameters in the model.

\subsection{Temporal structure of spike trains}

Although our model is quite detailed, including statistics of bursting, oscillatory modulation, and other spike train properties, we wanted to estimate how closely some model spike trains resemble their corresponding, real, spike trains recorded from cat visual cortex. We considered data recorded from an anaesthetized cat on two experimental sessions: one without or poor oscillatory responses (col11b44; see Fig. 3) and one with strong oscillations (col11b68; see Fig. 3) in the betahigh frequency band. Since the experimental trials were rather short (6.5 s) we selected, from one single electrode, multi-unit activity (MUA) such as to yield sufficient number of spikes when computing single-trial statistics (see next). We also chose a stimulation condition (center-surround stimulus, with a small sinusoidal grating placed in the center and a larger one in the surround) for which the MUA showed strong oscillatory behavior in col11b68 session but not in col11b44. For this stimulation condition, we obtained 20 experimental trials in both sessions. Next, for each experimental trial, one corresponding model trial (spike train) was generated. We computed the ISI distributions of spikes within bursts (burst ISI), the burst spike-count distributions, and the firing rate on the experimental trial, and plugged these parameters into the model, producing an artificial spike train having the same statistics (see Methods). Importantly, we wanted to model each trial independently such that the temporal structure of firing is maximally similar to the corresponding recorded trial. Statistics are thus computed separately for each trial. For oscillatory spike trains, the oscillation frequency was computed on the whole stimulation condition and plugged into each model trial (because the oscillation frequency was very stable across trials in the analyzed dataset). Next, the autocorrelation histograms (ACH) per stimulation condition were computed by averaging the ACHs computed on each of the 20 trials. The inter-spike interval (ISI) distributions were also computed.

In Figs. 3A and 3B we show that the ACHs and ISI distributions of the real and model spike trains were remarkably similar, both for the non-oscillatory and oscillatory case. This indicates that statistically, the real and modeled spike trains had similar local structure. At a first look, one could say that the model spike train can successfully replace the real one. To get further insight into this hypothesis, we computed the cross-correlation histograms $(\mathrm{CCH})$ between the 

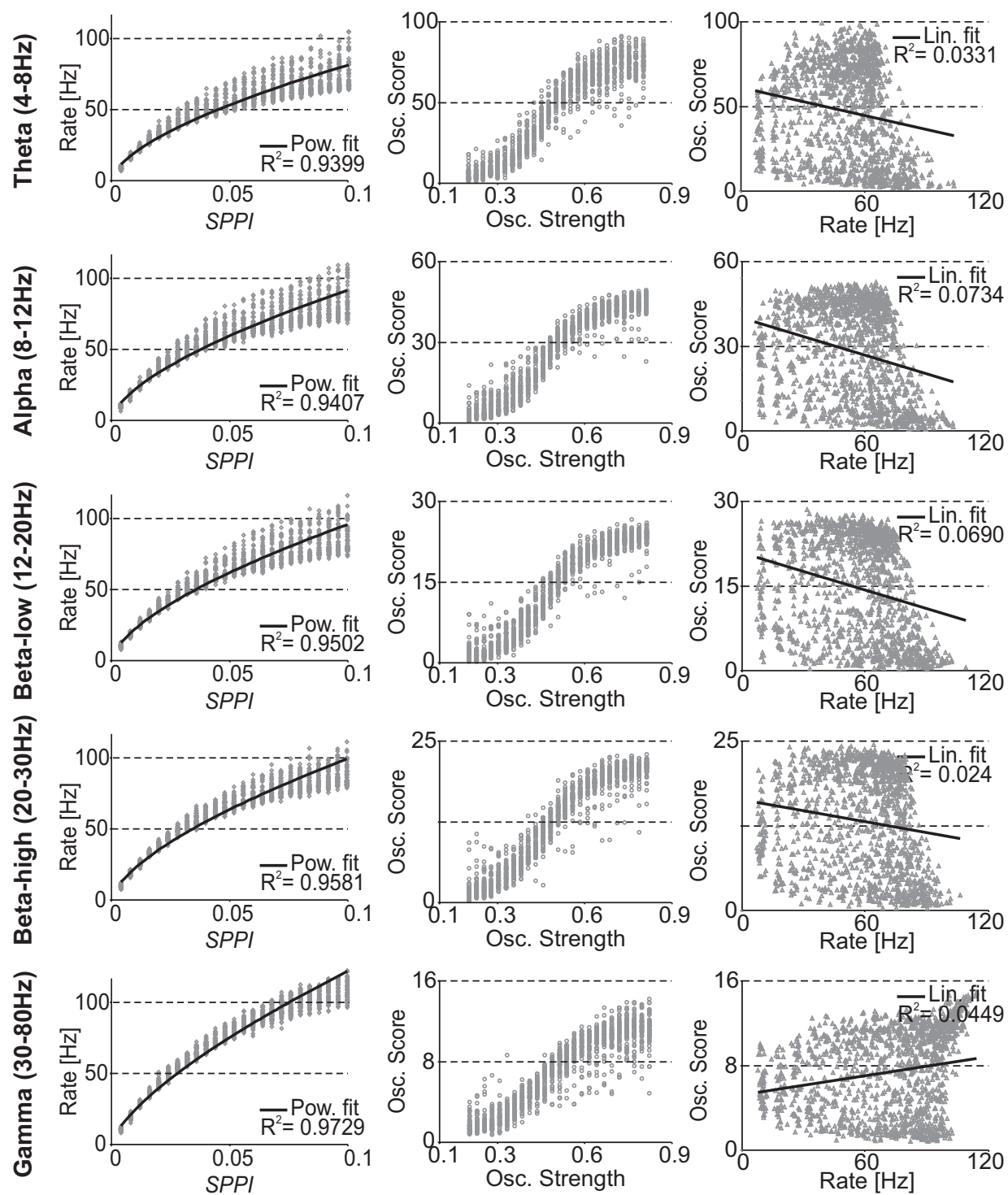

Fig. 2. Control of rate and oscillation strength. On the left column, scatter plots show the dependence of firing on the spike-probability-positive-integral: SPPI (5). The black line shows a power fit. The center column depicts the dependency of the measured oscillation score on the oscillation strength parameter, o, in the model (1). In the right column, scatter plots of the rate and oscillation score show no dependency between these two, as indicated by linear fits (black lines). 

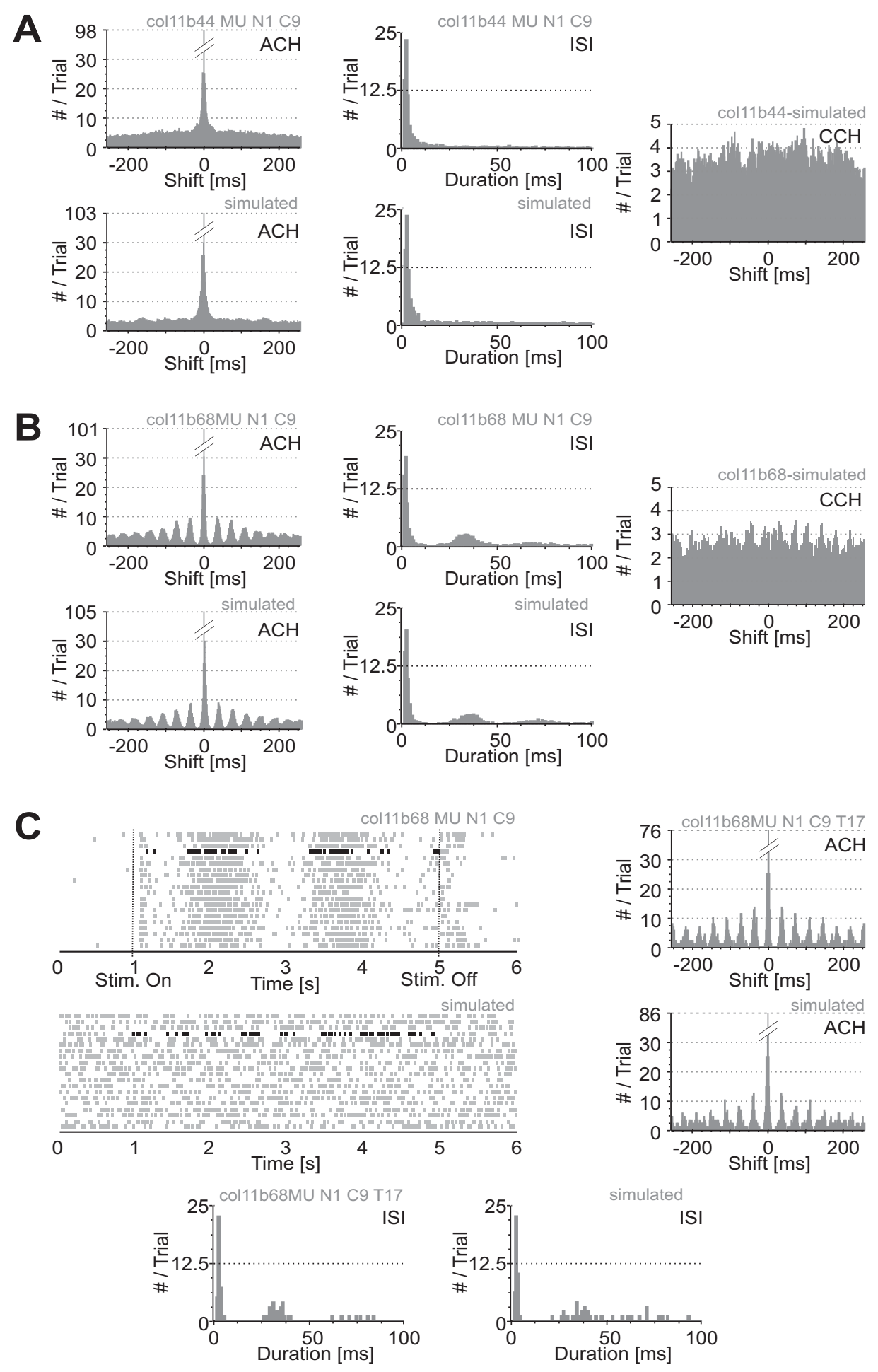
Fig. 3. Comparison between recorded and simulated spike trains. A: Non oscillatory data. The first row shows the ACH (left panel) and the ISI distribution (center panel) for non-oscillatory data. On the second row, the ACH (left panel) and ISI for simulated data (center panel) are shown. Note that the ACH and ISI plots are averages across 20 trials of the same condition. On the right panel the average cross-correlation between each recorded and artificial spike train is shown. B: The same plots as in A are shown for oscillatory data. The similarity of ACHs and ISI distributions between the recorded and simulated data is again clear. C: Differences between the recorded and simulated spike trains. The spikes are shown on the left: recorded data (upper traces, 20 consecutive trials) and simulated data (lower traces). For one recorded spike train and for its artificial model (both depicted in black) the ACHs (right panels) and ISI distributions (bottom panels) are shown. For each plot depicting recorded data the dataset, neuron, and condition labels are shown above the graph; for simulated data "simulation" is shown.

model and their corresponding, real, spike trains. Ideally, if the model successfully replaces the real spike train, the $\mathrm{CCH}$ should be very similar to the $\mathrm{ACHs}$ of both the real and modeled spike trains. This was, however, not the case. For non-oscillatory spike trains (Fig. 3A) the $\mathrm{CCH}$ was flat, without a peak, indicating that there was no consistent relationship (fine temporal correlation) between real and modeled spike timings. The oscillatory case was somewhat better, such that weak oscillatory modulation could be seen in the CCH (Fig. 3B), but the central peak was missing here as well. These results suggest that, although the statistical properties of the original spike trains are faithfully reproduced, the temporal dynamics of the trials are not. We show in Fig. 3C that, for a given spike train and its model, the ACHs and ISI distributions had reasonably close structure, but the location of spikes within the real and model trials was strikingly different. In the real spike train there was a clear modulation of the firing by the drifting sinusoidal grating stimulus. In the modeled spike train, the firing events were more or less arbitrarily located, despite the fact that global ISI statistics and ACHs structure were accurately reproduced (Fig. 3C).

\section{Discussion}

We have shown that the proposed spike train model allows one to independently control firing rate and oscillation strength. Thus, the spike train model can isolate reasonably well the two processes such that one can use it to calibrate data analysis methods which need to separate the effects of the firing rate from those of the oscillation strength [16].

Moreover, our results indicate that the model can reproduce the statistical properties of the real data quite faithfully. The ACHs and the ISI distributions of the model were strikingly similar to those of the real data and thus, we validated the model as being appropriate for studying properties of spike trains in terms of their ACHs. On the other hand, we have found that reproducing the statistics of neuronal firing can not account for the dynamics of the spike trains. There 
are multiple conclusions that stem from the above. First, given the complexity required by our model in order to reproduce realistic spike trains, we infer that simple, homogenous or inhomogeneous Poisson processes are crude and largely inappropriate approximations of cortical firing. These two types of processes need to be complemented by other, more complex ones, as real spike trains are very hard to model. One needs to add many constraints to the model and these constraints vary across neurons, stimuli, cortical states, and so forth. There is no universal 'recipe' for generating spike-trains, since neuronal behavior can be very rich and highly variable.

Second, modeling the fine temporal structure of spike trains, including their temporal dynamics can become very difficult because one needs to know the underlying drive received by neurons from the sensory (thalamic) and cortical inputs. An important implication of this fact is that collective coding strategies (where multiple, simultaneous spike trains are analyzed) cannot be easily studied with model spike trains. The reason is that, for collective codes, the relative dynamics of different neurons plays a crucial role. Reproducing the statistical properties of each individual spike train is obviously not enough. One could use a hybrid approach and also measure some instantaneous firing probability over time, but it is doubtful that the exact spike times can be accurately reproduced. Some have been able to achieve accurate modeling of firing processes, but only when the visual stimulus is known and mostly for early processing stages, such as the retina or LGN [18].

Finally, we want to emphasize that it is always important to properly assess the usefulness of a given spike train model. Models can prove useful for developing and calibrating data analysis methods [16], or for studying and quantifying given properties of the data [18]. In general however, the spiking behavior of neurons is both complex and variable. One needs to judge carefully which model captures the interesting properties relevant for a given scientific question. Furthermore, one has to be aware that, even if statistical properties of neuronal firing are precisely reproduced, the exact spiking dynamics stem from the complex interactions with other neurons and the drive from external stimuli. When one needs to generate a spike train that is very close to the original, the best model for the spike train is probably the spike train itself.

\section{Appendix}

To generate the data for Fig. 2 the following parameters were used: for $f_{o}(t)$, $\tau=0.5 * 1, m=0.5$ and $f_{o}(t)$ was allowed to deviate from the target oscillation frequency with at most $1 \mathrm{~Hz}$; for $f_{b}(t), \tau=0.1, m=3$ and $f_{b}(t)$ was bounded between 0 and $100 \mathrm{~Hz}$. Note that $\tau$ is given relative to the period of the target oscillation frequency. The oscillation strength, $o$, took values from 0.2 to 0.8 in 24 steps, SPPI was varied between 0.004 to 0.1 in 24 steps and the off set was set to 0.5. To generate the artificial data presented in Fig. 3, some of the parameters given above had to be adapted to each recorded spike train (trial) that was modeled. 


\section{Acknowledgments}

The authors acknowledge three grants of the Romanian Government (Human Resources Program RP-5/2007 contract 1/01.10.2007 and Ideas Program ID_48/2007 contract 204/01.10.2007 both financed by MECT/UEFISCSU, and Partnerships Program contract 11039/18.09.2007 financed by MECT/ANCS), a grant for the "Max Planck - Coneural Partner Group", and a Deutsche Forschungsgemeinschaft grant (NI 708/2-1).

\section{References}

1. Braitenberg, V., Schz, A.: Cortex: Statistics and Geometry of Neuronal Connectivity. 2nd edn, Springer, Berlin (1998)

2. Churchland, Patricia S., Sejnowski, T.J.: The Computational Brain. MIT Press, Cambridge MA (1999)

3. Destexhe, A., Rudolph, M., Par, D.: The high-conductance state of neocortical neurons in vivo. Nat. Rev. Neurosci. 4 (2003) 739-751

4. Tuckwell, H.C.: Stochastic Processes in the Neurosciences. Society for Industrial and Applied Mathematics, Philadelphia PA (1989)

5. Rieke, F., Warland, D., de Ruyter van Steveninck, R. and Bialek, W.: Spikes: Exploring the Neural Code. MIT Press, Cambridge MA (1997)

6. Dayan, P., Abbott, L.F.: Theoretical Neuroscience. MIT Press, Cambridge MA (2001)

7. Heeger, D.: Poisson model of spike generation. http://www.cns.nyu.edu/ david/handouts /poisson.pdf (2000)

8. Waters, J., Helmchen F.: Background synaptic activity is sparse in neocortex. J. Neurosci. 26(32) (2006) 8267-8277

9. Tiesinga, P., Fellous, J.M., Sejnowski, T.J.: Regulation of spike timing in visual cortical circuits. Nat. Rev. Neurosci. 9 (2008) 97-109

10. Evarts, E.V.: Temporal patterns of discharge of pyramidal tract neurons during sleep and waking in the monkey. J. Neurophysiol. 27 (1964) 152-171

11. Soltesz, I.: Diversity in the neuronal machine. Oxford University Press, New York (2005)

12. Kara, P., Reinagel, P., Reid, R.C.: Low response variability in simultaneously recorded retinal, thalamic, and cortical neurons. Neuron 27 (2000) 635-646

13. Steriade, M., McCormick, D.A., Sejnowski, T.J.: Thalamocortical oscillations in the sleeping and aroused brain. Science 262 (1993) 679-685

14. Steriade, M., Timofeev, I., Dürmüller, N., Grenier, F.: Dynamic properties of corticothalamic neurons and local cortical interneurons generating fast rhythmic (30-40 Hz) spike bursts. J. Neurophysiol. 79 (1998) 483-490

15. Buzsáki, G.: Rhythms of the brain. Oxford University Press, Oxford (2006)

16. Mureşan, R.C., Jurjut, O.F., Moca, V.V., Singer, W., Nikolić, D.: The Oscillation Score: An Efficient Method for Estimating Oscillation Strength in Neuronal Activity. J. Neurophysiol. 99 (2008) 1333-1353

17. DeBusk, B.C., DeBruyn, E.J., Snider, R.K., Kabara, J.F., Bonds, A.B.: Stimulus-dependent modulation of spike burst length in cat striate cortical cells. J. Neurophysiol. 78 (1997) 199-213

18. Keat, J., Reinagel, P., Reid, R.C., Meister, M.: Predicting Every Spike: A Model for the Responses of Visual Neurons. Neuron 30 (2001) 803-817 